\title{
Intravenous Ketamine Alleviates Pain in a Rheumatoid Arthritis Patient With Comorbid Fibromyalgia
}

\author{
Ashraf F. Hanna ${ }^{a}$, b, Bishoy Abraham ${ }^{a}$, Andrew Hanna ${ }^{a}$, \\ Micheal McKenna ${ }^{a}$, Adam J. Smitha
}

\begin{abstract}
A 49-year-old woman with rheumatoid arthritis (RA) and comorbid fibromyalgia presented to the clinic in extreme pain, described to be a 10 on an 11-point pain intensity numerical rating scale (PI-NRS). Because the patient also met the diagnostic criteria for fibromyalgia and conventional medications did not achieve adequate pain control for either condition, the off-label use of intravenous (IV) ketamine was presented as an alternative therapeutic option. The patient underwent 10 consecutive IV infusion sessions with increasing doses of ketamine hydrochloride without any complications. Following the 10th infusion, the patient reported that her pain was virtually nonexistent (PI-NRS 0 - 1).
\end{abstract}

Keywords: IV ketamine; Chronic pain; Pain management; Autoimmune disorders; NMDA antagonist

\section{Introduction}

Rheumatoid arthritis (RA) is a chronic inflammatory autoimmune disease that leads to stiffness, pain and swelling of the joints. Management goals include preventing further joint damage and disability, which requires arresting disease progress. To that end, numerous pharmacotherapies are currently available that target pathogenic cytokines such as TNF- $\alpha$, IL-1 $\beta$, or IL-6 [1]. These include five main classes of drugs: analgesics, non-steroidal anti-inflammatories (NSAIDs), glucocorticoids, non-biologic and biologic disease-modifying anti-rheumatic drugs [2]. Unfortunately, many patients have a sub-optimal response to these drug options or experience medication-related side effects that necessitate exploring other options. Interestingly, patients with RA have been associated with an increased risk in developing fibromyalgia [3].

Fibromyalgia is a disorder with clinical manifestations that include widespread pain, tenderness, fatigue, sleep distur-

Manuscript submitted February 5, 2018, accepted February 13, 2018

aFlorida Spine Institute, Clearwater, FL, USA

${ }^{\mathrm{b}}$ Corresponding Author: Ashraf Hanna, Florida Spine Institute, 2250 Drew St., Clearwater, FL 33765, USA. Email: asmithphd@me.com

doi: https://doi.org/10.14740/jmc3017w bance, and psychological distress [4]. It is commonly regarded as the "prototypical central sensitivity syndrome" [5]. While its etiology is not fully understood, fibromyalgia is thought to result from abnormal central pain processing and increased central sensitivity that results in intense, widespread pain. Fibromyalgia is diagnosed using the 2010 American College of Rheumatology (ACR) criteria [6]: 1) Widespread pain index (WPI) $\geq 7$ and symptom severity (SS) scale score $\geq 5$ or WPI 3 - 6 and SS scale score $\geq 9$. 2) Symptoms have been present at a similar level for at least 3 months. 3) The patient does not have a disorder that would otherwise explain the pain.

A recent epidemiological study characterized the prevalence of fibromyalgia among patients without pain, with regional pain, and with widespread pain [4]. Using the ACR criteria for fibromyalgia [6], the study found that the estimated prevalence of fibromyalgia was $2.0 \%$ for both sexes, $3.4 \%$ for women and $0.4 \%$ for men. Interestingly, RA is widely known to be more common in women with epidemiological data indicating that the sex ratio is somewhere between 2:1 and 3:1 [7]. Thus, fibromyalgia and RA are significant and prevalent health problems that disproportionately affect women.

Ketamine is a long-known, dissociative anesthetic that strongly antagonizes the N-methyl-D-aspartate (NMDA) receptor. Although this is its most well-known pharmacological action, emerging research has shown that ketamine produces effects that reach far beyond NMDA antagonism. A recent clinical study investigated the immune response in patients undergoing elective abdominal surgery was given intravenous (IV) ketamine or a saline placebo prior to induction of general anesthesia [8]. Interestingly, the group of patients that had received ketamine showed a significantly suppressed production of IL-6 $(\mathrm{P}<0.01)$ compared to controls. Moreover, the ketamine group also produced less TNF- $\alpha$ post-operation. Numerous clinical studies have shown that ketamine possesses significant analgesic properties [9-11]. Our own clinical experience using ketamine for treatment-refractory pain has been positive and we have previously reported long-term pain relief in a patient with fibromyalgia [12].

Given these immunomodulatory, anti-inflammatory, and analgesic effects of ketamine, it seems to have tremendous potential for treating severe cases of RA that do not respond to conservative treatment options. Recently, the effects of ketamine in a rat model of RA were investigated and it was found that ketamine significantly reduced arthritis severity as indicated by reduced joint swelling, reduced infiltration of inflammatory cells and reduced joint destruction in the acute 


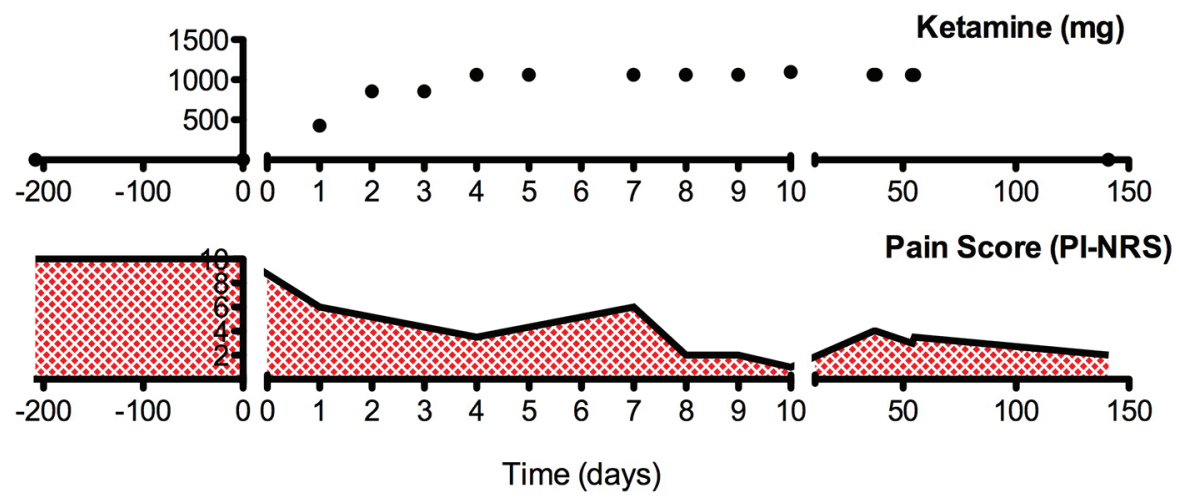

Figure 1. The summarization of the clinical course.

and chronic phase of arthritis [13]. Although these results were very promising, preclinical data do not always translate to the clinic and no clinical data were available for the use of ketamine in patients with RA.

The case report described herein will discuss the clinical course of a female patient with RA and comorbid fibromyalgia treated with IV ketamine.

\section{Case Report}

A 49-year-old woman was referred to the Florida Spine Institute (Clearwater, FL) by her rheumatologist after being diagnosed with seronegative erosive RA. Her diagnostic labs indicated that she had high levels of C-reactive protein but was negative for rheumatoid factor and anti-cyclic citrullinated peptide antibody. Under the care of her rheumatologist, her arthritis was refractory to several conventional treatment options including methotrexate, tocilizumab, abatacept, belimumab, apremilast, golimumab, and leflunomide. On January 27, 2016, her pain intensity was reported to be 10 on an 11-point pain intensity numerical rating scale (PI-NRS), where 0 is no pain and 10 is worst possible pain.

The patient described cervical bilateral symmetrical facetogenic pain and symmetric bilateral sacroiliac joint pain with stiffness in the mornings for more than $60 \mathrm{~min}$. Moreover, she had symmetric diffuse joint pain in bilateral proximal joints of the hands and shoulders. She also had diffuse muscular pain and met the diagnostic criteria for fibromyalgia established by the ACR [6]. Numerous conservative treatment approaches were tried including physical therapy for cervical and lumbar pain with mild and temporary relief. Trigger point injections and bilateral sacroiliac joint injections under fluoroscopy did not result in any significant pain relief. She also underwent cervical radiofrequency ablation with no relief from pain. Since other therapeutic options did not achieve adequate pain control, the off-label use of IV ketamine was discussed with the patient. Because ketamine can exacerbate schizophrenia and abnormal liver function, patients should be screened for these conditions prior to starting ketamine. The patient was scheduled for IV ketamine therapy after clearing a psychiatric evaluation for schizophrenia and passing a liver function test.
The patient started a 10-day IV ketamine infusion protocol on August 22, 2016 at the Florida Spine Institute. The starting dose was $428 \mathrm{mg}$ ketamine $\mathrm{HCl}$ in $100 \mathrm{~mL}$ of $0.9 \%$ sodium chloride. This was escalated to $856 \mathrm{mg}$ for the second and third infusions and further escalated to $1063 \mathrm{mg}$ for the remaining infusions. All doses were well tolerated and no unexpected adverse effects were observed.

The clinical course of this patient is illustrated in Figure 1. Following the first infusion session, the patient indicated that her pain intensity had fallen to 6 (PI-NRS). Following the last infusion, the patient indicated that her pain had been almost completely eliminated (PI-NRS 0 - 1). The patient also remarked that her RA symptoms had improved drastically and she was no longer experiencing joint pain and stiffness in the mornings. However, the patient returned for a 2-day booster infusion approximately 3 weeks later due to her pain levels returning to $40 \%$ of her pre-ketamine levels. Approximately 3 months after this booster infusion, the patient returned for another 2-day booster infusion with pain levels at 30-40\% of her pre-ketamine levels. At the most recent follow-up visit ( 90 days since the last booster infusion), the patient reported that her pain levels were low (PI-NRS 2), an $80 \%$ improvement from her pre-ketamine levels.

\section{Discussion}

To our knowledge, this is the first report on the use of ketamine in a patient with RA. Immediately following the 10-day ketamine infusion protocol, the patient's pain was alleviated completely but did eventually return to $20-40 \%$ of pre-ketamine levels necessitating additional 2-day booster infusions. It is possible that a take-home ketamine-containing drug product may more adequately maintain the results achieved immediately following the 10-day infusion. However, there are currently no FDA-approved take-home ketamine products that can be prescribed. Overall, the patient was satisfied with the treatment outcome and experienced no adverse events. Because repeat exposures to ketamine have been associated with hepatotoxicity, regular blood tests are merited to monitor the patient's liver enzymes (alanine transaminase, alkaline phosphatase, aspartate transaminase, and $\gamma$-glutamyl transferase) 
[14]. This patient never exhibited signs of hepatotoxicity with normal labs pre-infusion and at 24 and 56 days after the first infusion.

RA requires therapeutic options that can address the causal autoimmune component of the disease while also producing adequate analgesia for cases that are associated with severe pain. Although some therapeutic options already exist for $\mathrm{RA}$, it is important to note that they are not always effective. Moreover, patients with comorbid diseases such as fibromyalgia often do not achieve adequate pain control with existing therapies and are frequently prescribed increasing doses of potent opioid analgesic medications. Ketamine appears to possess unique immunomodulatory and analgesic properties that effectively attenuate inflammation and reduce pain without the use of opioid/NSAID analgesics - which have become a worldwide epidemic leading to addiction, overdose, and death [15]. Moreover, our own clinical experience and published clinical studies by others [16] have found that ketamine can be an opioid-sparing option that can be used as a tool to reduce and, in some cases, discontinue chronic opioid use.

\section{Conclusion}

Conclusions derived from the clinical evidence described in this case report are limited in that it involves the experience of only one patient. Moreover, as a case report, there was no placebo control. Since ketamine is already an FDA-approved drug with decades of safe human experience, there is considerably less risk for pharmaceutical companies willing to pursue new indications with patient-friendly routes of administration. We are hopeful that future adequately powered and placebocontrolled clinical trials may confirm that ketamine is safe and effective for the treatment of autoimmune diseases such as RA.

\section{Conflict of Interest}

The authors declare no conflict of interest.

\section{Abbreviations}

RA: rheumatoid arthritis; IV: intravenous; FDA: United States Food and Drug Administration; PI-NRS: pain intensity numerical rating scale; NSAIDs: non-steroidal anti-inflammatories; ACR: American College of Rheumatology; WPI: widespread pain index; SS: symptom severity; NMDA: N-methyl-D-aspartate

\section{References}

1. Furst DE, Emery P. Rheumatoid arthritis pathophysiology: update on emerging cytokine and cytokine-associated cell targets. Rheumatology (Oxford). 2014;53(9):15601569.

2. Kumar P, Banik S. Pharmacotherapy options in rheuma- toid arthritis. Clin Med Insights Arthritis Musculoskelet Disord. 2013;6:35-43.

3. Wolfe F, Hauser W, Hassett AL, Katz RS, Walitt BT. The development of fibromyalgia-I: examination of rates and predictors in patients with rheumatoid arthritis (RA). Pain. 2011;152(2):291-299.

4. Wolfe F, Ross K, Anderson J, Russell IJ, Hebert L. The prevalence and characteristics of fibromyalgia in the general population. Arthritis Rheum. 1995;38(1):19-28.

5. Boomershine CS. Fibromyalgia: the prototypical central sensitivity syndrome. Curr Rheumatol Rev. 2015;11(2):131-145.

6. Wolfe F, Clauw DJ, Fitzcharles MA, Goldenberg DL, Katz RS, Mease P, Russell AS, et al. The American College of Rheumatology preliminary diagnostic criteria for fibromyalgia and measurement of symptom severity. Arthritis Care Res (Hoboken). 2010;62(5):600-610.

7. Alamanos Y, Drosos AA. Epidemiology of adult rheumatoid arthritis. Autoimmun Rev. 2005;4(3):130-136.

8. Beilin B, Rusabrov Y, Shapira Y, Roytblat L, Greemberg L, Yardeni IZ, Bessler H. Low-dose ketamine affects immune responses in humans during the early postoperative period. Br J Anaesth. 2007;99(4):522-527.

9. Schwartzman RJ, Alexander GM, Grothusen JR, Paylor T, Reichenberger E, Perreault M. Outpatient intravenous ketamine for the treatment of complex regional pain syndrome: a double-blind placebo controlled study. Pain. 2009;147(1-3):107-115.

10. Aida S, Yamakura T, Baba H, Taga K, Fukuda S, Shimoji K. Preemptive analgesia by intravenous low-dose ketamine and epidural morphine in gastrectomy: a randomized double-blind study. Anesthesiology. 2000;92(6):16241630.

11. Eide PK, Jorum E, Stubhaug A, Bremnes J, Breivik H. Relief of post-herpetic neuralgia with the N-methyl-D-aspartic acid receptor antagonist ketamine: a double-blind, cross-over comparison with morphine and placebo. Pain. 1994;58(3):347-354.

12. Hanna AF, Smith AJ. Intravenous ketamine produces long-term pain relief in a patient with fibromyalgia. Fibromyalgia: Open Access. 2016;1(1).

13. Boettger MK, Weber K, Gajda M, Brauer R, Schaible HG. Spinally applied ketamine or morphine attenuate peripheral inflammation and hyperalgesia in acute and chronic phases of experimental arthritis. Brain Behav Immun. 2010;24(3):474-485.

14. Noppers IM, Niesters M, Aarts LP, Bauer MC, Drewes AM, Dahan A, Sarton EY. Drug-induced liver injury following a repeated course of ketamine treatment for chronic pain in CRPS type 1 patients: a report of 3 cases. Pain. 2011;152(9):2173-2178.

15. Manchikanti L, Helm S, 2nd, Fellows B, Janata JW, Pampati V, Grider JS, Boswell MV. Opioid epidemic in the United States. Pain Physician. 2012;15(3 Suppl):ES9-38.

16. Kim SH, Kim SI, Ok SY, Park SY, Kim MG, Lee SJ, Noh $\mathrm{JI}$, et al. Opioid sparing effect of low dose ketamine in patients with intravenous patient-controlled analgesia using fentanyl after lumbar spinal fusion surgery. Korean J Anesthesiol. 2013;64(6):524-528. 